\title{
Identifying the Food Sources of Selected Minerals for the Adult European Population among Rice and Rice Products
}

\author{
Joanna Bielecka, Renata Markiewicz-Żukowska *, Patryk Nowakowski (D), Anna Puścion-Jakubik (D), \\ Monika Grabia D, Anita Mielech D, Jolanta Soroczyńska and Katarzyna Socha $\mathbb{D}$
}

check for updates

Citation: Bielecka, J.; MarkiewiczŻukowska, R.; Nowakowski, P.; Puścion-Jakubik, A.; Grabia, M.; Mielech, A.; Soroczyńska, J.; Socha, K. Identifying the Food Sources of Selected Minerals for the Adult European Population among Rice and Rice Products. Foods 2021, 10, 1251. https://doi.org/10.3390/ foods10061251

Academic Editors: Agostinho

Almeida, Edgar Pinto and

Cristina Couto

Received: 10 May 2021

Accepted: 28 May 2021

Published: 31 May 2021

Publisher's Note: MDPI stays neutral with regard to jurisdictional claims in published maps and institutional affiliations.

Copyright: (c) 2021 by the authors. Licensee MDPI, Basel, Switzerland. This article is an open access article distributed under the terms and conditions of the Creative Commons Attribution (CC BY) license (https:/ / creativecommons.org/licenses/by/ $4.0 /)$.
Department of Bromatology, Medical University of Białystok, Mickiewicza 2D Street, 15-222 Białystok, Poland; joanna.bielecka@umb.edu.pl (J.B.); patryk.nowakowski@umb.edu.pl (P.N.); anna.puscion-jakubik@umb.edu.pl (A.P.-J.); monika.grabia@umb.edu.pl (M.G.); anita.mielech@umb.edu.pl (A.M.); jolasor@interia.pl (J.S.); katarzyna.socha@umb.edu.pl (K.S.)

* Correspondence: remnar@poczta.onet.pl; Tel.: +48-85-748-5469

\begin{abstract}
The problem of dietary deficiency of several essential elements among different stages of life is still observed. The consumption of whole grains (among others unprocessed rice) is recommended as a part of a healthy diet. This research aimed to determine the content of selected macro- and microelements in rice and rice products to verify whether the tested products could be regarded as a source of selected minerals in the diet of the adult European population. Methods: A total of 99 samples from 12 groups of rice products (basmati, black, brown, parboiled, red, wild, white rice and expanded rice, rice flakes, flour, pasta, and waffles) were obtained. The atomic absorption spectrometry method (AAS) was used to determine the content of $\mathrm{Ca}, \mathrm{Cu}, \mathrm{Fe}, \mathrm{Mg}$, $\mathrm{Mn}$, Se and $\mathrm{Zn}$ in the study material. Results: The average measured contents of $\mathrm{Ca}, \mathrm{Cu}, \mathrm{Fe}, \mathrm{Mg}, \mathrm{Mn}$, Se and $\mathrm{Zn}$ were as follows: $226.3 \pm 160.6 \mathrm{mg} / \mathrm{kg}, 3.6 \pm 2.8 \mathrm{mg} / \mathrm{kg}, 9.4 \pm 7.0 \mathrm{mg} / \mathrm{kg}, 618.0 \pm 498.4 \mathrm{mg} / \mathrm{kg}$, $16.7 \pm 10.0 \mathrm{mg} / \mathrm{kg}, 242.9 \pm 140.4 \mu \mathrm{g} / \mathrm{kg}$ and $19.5 \pm 15.0 \mathrm{mg} / \mathrm{kg}$, respectively. Statistical analyses confirmed the differences in the levels of the studied elements between the subgroups of processed and unprocessed products. Considering the tolerable upper intake level of studied elements, the tested products could be regarded as safe to consume. Conclusion: All tested products can be recommended as a source of $\mathrm{Cu}, \mathrm{Mn}$, and Se, while a majority of studied products can be considered a source of $\mathrm{Mg}$ and $\mathrm{Zn}$ in the diet of the adult European population.
\end{abstract}

Keywords: trace elements; nutrients; rice; rice products; dietary sources; human health

\section{Introduction}

The consumption of whole grains (unprocessed maize, oats, wheat and unpolished rice) is recommended as a part of a healthy diet. According to the Food and Agriculture Organization (FAO, Geneva, Switzerland) statistics, in 2020, global cereal production reached 2.7 billion tonnes for which rice was the second-largest crop after wheat (764.9 and $509.1 \mathrm{mln}$ of tonnes, respectively). The predominant part of the rice available on the world market is produced in Asian countries ( $>90 \%$ ); only $0.6 \%$ is cultivated in European countries. The world's three top producers of rice include China followed by India and Indonesia. The highest average rice consumption between 2016 and 2018 in Asian and Pacific countries was estimated at $77.6 \mathrm{~kg}$ per capita per year, while the lowest was in the European countries with $5.8 \mathrm{~kg}$ per capita per year [1].

Rice is one of the most important crops considering the total production as well as consumption worldwide. Two rice species-Oryza sativa S. (Asian rice) and Oryza glaberrima L. (African rice)-are cultivated. However, O. sativa is the predominant species, grown across the world; in turn, O. glaberrima is cultivated mainly in Africa and differs only slightly in its morphological aspects [2]. In the structure of paddy rice grains, two main parts can be specified: the hull and the caryopsis. The hull located outside the grain is rich in minerals and constitutes about $20 \%$ of the whole grain. The caryopsis is under the hull 
and includes the bran layer, endosperm and germ. The germ is the most nutritious part, rich in protein, fatty acids and mineral compounds [3]. Depending on the processes carried out on the grain, the rice can be classified as paddy (kernels are present inside the hull), brown (when the caryopsis is removed in the hulling process), white or milled (the milling process removes the bran layer and the germ from the brown rice) or parboiled (soaked in hot water then steamed before drying) [4]. White rice has a lower content of protein (especially a reduced lysine amount), minerals and fiber in comparison to brown rice. The parboiling process reduces the glycemic index and additionally contributes to the transfer of some minerals into the grain, which has a positive effect on its nutritional value [4].

It is observed that high consumption of white rice is related to increased risk of impaired glucose homeostasis and the occurrence of type 2 diabetes mellitus (T2DM). On the other hand, black rice intake is related to a lower postprandial blood glucose response (the glycemic index) and consequently, reduced risk of metabolic disorders, such as obesity, hyperglycemia, dyslipidemia, T2DM and hypertension. The anti-cancer activity of black rice bioactive compounds has also been demonstrated [4].

Well-balanced nutrition should provide not only adequate amounts of essential nutrients, such as proteins, carbohydrates and fats as well as vitamins and minerals. According to the available literature, the problem of unbalanced diets is still observed. The inadequate intake of macroelements such as calcium (Ca) or magnesium (Mg) was reported [5]. On the other hand, the over intake of copper $(\mathrm{Cu})$ and manganese $(\mathrm{Mn})$ were determined [6].

Ca plays a crucial role in the mineralization of bones and teeth, takes part in neuromuscular conduction and is required in maintaining the balance of body fluids within cells. Inadequate dietary intake of Ca may result in increased bone mass loss and osteoporotic fractures in old age [7].

$\mathrm{Cu}$ is essential for the functioning of several physiological pathways due to being a cofactor or structural component of many enzymes (e.g., cytochrome c oxidase, ceruloplas$\mathrm{min})$. $\mathrm{Cu}$ is important in the neuromodulation process, in response to low blood pressure as well as in angiogenesis. In the case of insufficient intake of $\mathrm{Cu}$ with diet, hematological manifestations (such as anemia), bone abnormalities or neurological symptoms may occur [8].

Iron $(\mathrm{Fe})$ is one of the key elements in hematopoiesis. Furthermore, Fe is important for oxygen transport in the human body and is an important element in several metabolic processes, such as the synthesis of deoxyribonucleic acid (DNA) and the transport of electrons. Fe deficiency may lead to the development of anemia, which adversely influences cognitive functions, learning ability and immunity responses. Anemia is particularly dangerous in pregnancy because it is related to several negative outcomes, such as low birth weight or maternal and perinatal mortality [9].

$\mathrm{Mg}$ is involved in more than 300 biochemical reactions in the body as a cofactor of enzymes. It takes part, among others, in the production of energy, neuromuscular conduction, bone mineralization, DNA and RNA synthesis. Inadequate Mg intake increases the risk of the development of numerous chronic diseases, such as insulin resistance, T2DM, hypertension, Alzheimer's disease or osteoporosis [10].

$\mathrm{Mn}$ is the next element, which participates in a variety of metabolic reactions in an organism. Mn is essential for the proper functioning of the immune, nervous and reproductive systems. Moreover, Mn plays a role in antioxidant responses, energy metabolism and inactivation of metalloenzymes and enzymes crucial for the synthesis of neurotransmitters. Insufficient Mn intake may be associated with impaired metabolism of proteins, fats and carbohydrates, growth disturbances and fertility problems. Excessive Mn intake may lead to the development of a neurodegenerative disorder, called manganism, which causes dopaminergic neuronal death [11].

Selenium (Se) is a trace element characterized by a narrow range between physiological status, deficiency and excessive (toxic) concentration. At the same time, Se is an important element in many physiological processes directly or indirectly. It constitutes a part of antioxidant enzymes (e.g., glutathione peroxidases), supports the response of the 
immune system and is required for maintaining reproductive health. Se is essential for proper thyroid functioning by being a part of iodotyrosine deiodinase, which catalyzes the deiodonization of thyroxine (T4) to triiodothyronine (T3). Considering the adverse health effects of inadequate Se intake, disorders of the heart muscle and joints are mainly observed. Increased risk of infertility and prostate cancer in men and neurological impairments are also possible [12].

Zinc (Zn) plays numerous roles in the human body, which could be classified as regulatory, catalytic and structural functions. Among the most important $\mathrm{Zn}$ functions, the effect on wound healing, supporting the immune and reproductive systems, regulation of blood pressure and heart rate as well as proper insulin secretion are described. Zn deficiency may impair the functioning of the immune, reproductive, nervous and gastrointestinal systems [13].

Because of the lack of favorable conditions to grow, rice species are not cultivated in Poland. Rice grains available on the Polish market are imported from different world regions, mainly from Asia, Southern Europe and Southern Africa. In this study, we were interested in the assessment of the nutritionally important components of which include macro- and microelements of different rice grains and rice products. To the best of our knowledge, such a broad group of different types of rice products has not yet been studied so far. We collected a broad range of diverse products, including seven rice subgroups and five subgroups of rice products.

In our research, we aimed to determine the content of selected macro- ( $\mathrm{Ca}$ and $\mathrm{Mg}$ ) and microelements $(\mathrm{Cu}, \mathrm{Fe}, \mathrm{Mn}, \mathrm{Se}$, and $\mathrm{Zn})$ in rice and rice products, which has not been investigated yet. Moreover, we estimated whether the tested products can be regarded as a food source of the mentioned elements in the diet of the adult European population.

\section{Materials and Methods}

\subsection{Sample Collection}

The samples, representative of the overall Polish market, were obtained from local markets in north-eastern Poland. A total of 99 products were acquired between March and May 2020. We collected twelve subgroups of products (at minimum five samples each), among which seven were different types of rice: basmati $(n=10)$, black $(n=6)$, brown $(n=10)$, parboiled $(n=10)$, red $(n=5)$, wild $(n=5)$, white $(n=11)$. Moreover, we also obtained five different types of rice products: expanded rice $(n=8)$, rice flakes $(n=12)$, flour $(n=6)$, pasta $(n=7)$, and waffles $(n=9)$. The samples were not duplicated; each one of the products in the subgroups (e.g., among flakes) was purchased from a different producer. Considering the country of origin, the lack of this information in 19 products was observed. Most of the samples collected were imported from Asian countries $(n=53)$, while 27 were imported from Europe.

\subsection{Sample Digestion}

The preparation of samples for the mineralization process included homogenization in the stainless-steel mill. The samples were not treated thermally prior to the analysis. An appropriate amount $(0.2-0.3 \mathrm{~g})$ was weighted and transferred into mineralization vessels, then $4 \mathrm{~mL}$ spectrally pure concentrated $(69 \%)$ nitric $(\mathrm{V})$ acid was added (Tracepur, Merck, Darmstadt, Germany). A close-loop system was used to perform microwave digestion (Berghof, Speedwave, Eningen, Germany).

\subsection{Analysis of Studied Elements Contents}

The content of selected elements in the digested rice samples was determined by atomic absorption spectrometry (AAS), using the Z-2000 instrument (Hitachi, Tokyo, Japan). Before the analysis, the majority of the mineralized samples were diluted 20 times for $\mathrm{Ca}$ and $\mathrm{Cu}, 5$ for $\mathrm{Fe}, 50$ for $\mathrm{Mg}$, 100 for $\mathrm{Mn}, 2$ for Se and 10 for $\mathrm{Zn}$. In the case of $\mathrm{Cu}, \mathrm{Mn}$, and Se, the flameless AAS technique with electrothermal atomization in a graphite cuvette was applied. To determine the Se content, the palladium-magnesium matrix modifier 
(Merck, Darmstadt, Germany) was added (Pd concentration: $1500 \mathrm{mg} / \mathrm{L} ; \mathrm{Mg}$ concentration: $900 \mathrm{mg} / \mathrm{L})$, while for the Mn measurement, magnesium nitrate $\left(\mathrm{Mg}\left(\mathrm{NO}_{3}\right)_{2}\right.$ concentration: $100 \mathrm{mg}$ /L, Sigma-Aldrich, Merck, Darmstadt, Germany) as a modifier was used. The flame AAS technique in the acetylene-air flame with Zeeman background correction for the determination of $\mathrm{Ca}, \mathrm{Fe}, \mathrm{Mg}, \mathrm{Zn}$ was used. Moreover, in the case of $\mathrm{Ca}$ and $\mathrm{Mg}, 1 \%$ lanthanum chloride ( $\mathrm{LaCl}_{3}$, Sigma-Aldrich, Merck, Darmstadt, Germany) was adopted as a masking agent. The analytical conditions of the process of the flameless and flame techniques are presented in Table 1.

Table 1. The analytical conditions of flameless AAS technique ( $\mathrm{Cu}, \mathrm{Mn}$ and $\mathrm{Se})$ and flame AAS technique (Ca, Fe, Mg and $\mathrm{Zn}$ ) in determining the content of elements in rice samples.

\begin{tabular}{cccccccc}
\hline & \multicolumn{3}{c}{ Element } & & \\
\hline Parameter & Cu & Mn & Se & Ca & Fe & Mg & Zn \\
\hline Wavelength $(\mathrm{nm})$ & 324.8 & 279.5 & 196.0 & 422.7 & 248.3 & 285.2 & 213.9 \\
Lamp current $(\mathrm{mA})$ & 7.5 & 7.5 & 14.5 & 7.5 & 12.5 & 7.5 & -5.5 \\
Drying $\left({ }^{\circ} \mathrm{C}\right)$ & $80 / 140$ & $80 / 140$ & $70 / 100$ & - & - & - & - \\
Ashing $\left({ }^{\circ} \mathrm{C}\right)$ & $600 / 600$ & $750 / 750$ & $600 / 600$ & - & - & - & - \\
Atomization $\left({ }^{\circ} \mathrm{C}\right)$ & $2400 / 2400$ & $2300 / 2300$ & $2700 / 2700$ & - & - & - \\
Cuvette cleaning $\left({ }^{\circ} \mathrm{C}\right)$ & $2500 / 2500$ & $2500 / 2500$ & $2800 / 2800$ & - & - & - \\
\hline
\end{tabular}

\subsection{Method Validation}

The certified reference materials (CRMs) were used to control the quality of performed analyses (Table 2). In the case of $\mathrm{Ca}, \mathrm{Cu}, \mathrm{Fe}, \mathrm{Mg}$, $\mathrm{Mn}$, and $\mathrm{Zn}$, corn flour (INCT-CF-3) was applied, while for Se, mushroom powder (CS-M-3) was used (both standards produced by the Institute of Nuclear Chemistry and Technology, Warsaw, Poland). The CRMs were analyzed before and for every ten determinations.

Table 2. The results obtained in the method validation.

\begin{tabular}{ccccc}
\hline Element & $\begin{array}{c}\text { Detection Limit } \\
\text { for Method * }\end{array}$ & $\begin{array}{c}\text { Detection Limit } \\
\text { for Samples }\end{array}$ & $\begin{array}{c}\text { Recovery for } \\
\text { CRM }\end{array}$ & Precision (\%) \\
\hline $\mathbf{C a}$ & $0.10 \mathrm{mg} / \mathrm{L}$ & $9.26 \mathrm{mg} / \mathrm{kg}$ & 98.4 & 3.6 \\
$\mathbf{C u}$ & $0.65 \mu \mathrm{g} / \mathrm{L}$ & $0.24 \mathrm{mg} / \mathrm{kg}$ & 99.2 & 2.1 \\
$\mathbf{F e}$ & $0.11 \mathrm{mg} / \mathrm{L}$ & $1.29 \mathrm{mg} / \mathrm{kg}$ & 98.7 & 2.5 \\
$\mathbf{M g}$ & $0.009 \mathrm{mg} / \mathrm{L}$ & $3.33 \mathrm{mg} / \mathrm{kg}$ & 98.8 & 2.7 \\
$\mathbf{M n}$ & $0.14 \mu \mathrm{g} / \mathrm{L}$ & $0.13 \mathrm{mg} / \mathrm{kg}$ & 101.2 & 2.4 \\
$\mathbf{S e}$ & $1.55 \mu \mathrm{g} / \mathrm{L}$ & $57 \mu \mathrm{gg} / \mathrm{kg}$ & 97.5 & 4.7 \\
$\mathbf{Z n}$ & $0.015 \mathrm{mg} / \mathrm{L}$ & $1.39 \mathrm{mg} / \mathrm{kg}$ & 100.9 & 1.8 \\
\hline
\end{tabular}

* Estimated as a characteristic concentration in standard solution for 0.0044 absorbance. ${ }^{1}$ Detection limit considering amount weighted and diluted for samples.

\subsection{Assessment Whether Tested Products Could Be Regarded as a Source of Studied Nutrients}

We assessed whether the tested groups of products contained in $100 \mathrm{~g}$, at minimum, $15 \%$ of the reference value intake (RVI) established by the European Parliament and of the Council. If this requirement was met, the product could be regarded as a source of the studied elements for the adult European population [14].

\subsection{Estimation of the Risk of Adverse Health Effects}

Based on the results obtained in this research and the tolerable upper intake level (TUIL) determined by the National Institutes of Health in the United States of America, the risk of adverse health effects resulting from daily consumption of the studied nutrients was evaluated. The limits considered the highest daily nutrient intake, which should pose no adverse health effects to almost all individuals in the general population. The following levels for adults over 19 years of age were established: $\mathrm{Cu}, 10 \mathrm{mg} /$ day; $\mathrm{Fe}, 45 \mathrm{mg} /$ day; 
$\mathrm{Mn}, 11 \mathrm{mg} /$ day; Se, $400 \mu \mathrm{g} /$ day; and $\mathrm{Zn}, 40 \mathrm{mg} /$ day. In the case of Ca, two age groups were specified: $2500 \mathrm{mg} /$ day (for adults aged 19-50 years) and $2000 \mathrm{mg} /$ day (for adults over 50 years). For $\mathrm{Mg}$, the TUIL was given for pharmacological agents use and did not include intake from food and water; therefore, it was not analyzed in our research [15]. The maximum amounts of safe intake levels of studied products were calculated.

\subsection{Statistical Analyses}

Statistica software (Tibco, Palo-Alto, CA, U.S.A.) was used to analyze the data. The normality distribution of the data was checked by the Shapiro-Wilk test. The results were shown as mean $(\mathrm{X})$ with standard deviation (SD), minimum (Min), maximum (Max) as well as the median and interquartile range (lower quartile $Q_{1}$, upper quartile $Q_{3}$ ). KruskallWallis Analysis of Variance (ANOVA) with post-hoc analysis to compare the content of the studied elements between the subgroups of products was performed. Significant differences were assumed at $p$-values $<0.001,<0.01$ and $<0.05$.

\section{Results}

The results obtained in our analysis are presented in Tables 3 and 4 .

None of the subgroups tested can be considered a source of Ca and Fe in the diet of the adult European population (the RVI was lower than 15\%). Contrastingly, every studied subgroup could be taken into account as a source of $\mathrm{Cu}, \mathrm{Mn}$, and $\mathrm{Se}$. In the case of $\mathrm{Mg}$, six subgroups (basmati, expanded, parboiled, white rice, flakes and pasta) and three of $\mathrm{Zn}$ (basmati, parboiled rice and pasta) did not meet the requirements; therefore, they cannot be regarded as a source of these nutrients in the diet (Tables 3 and 4).

The TUIL for Ca in every subgroup was higher than $7000 \mathrm{~g} /$ day, while that for Fe was higher than $2570 \mathrm{~g} /$ day. TUIL for Cu ranged from $811 \mathrm{~g} /$ day for wild rice to $4579 \mathrm{~g} /$ day for white rice; for $\mathrm{Mn}$, it was lowest for red rice (298 g/day) and highest for parboiled rice $(5300 \mathrm{~g} /$ day). In the case of Se, the lowest TUIL was calculated for parboiled rice ( $1297 \mathrm{~g} /$ day) and at the same time, the highest ( $2321 \mathrm{~g} /$ day) for wild rice. The TUIL for $\mathrm{Zn}$ ranged from $672 \mathrm{~g} /$ day for wild rice to $7265 \mathrm{~g}$ /day for parboiled rice.

The statistical analysis confirmed the differences in the content of studied elements between the unprocessed (e.g., black or red rice) and processed (e.g., flakes or pasta) subgroups of products. The Kruskall-Wallis Analysis of Variance (ANOVA) test with post-hoc analysis was used. Only in the case of $\mathrm{Cu}$, no differences were found between the studied products. The differences between the contents of the studied elements in the studied product groups are shown in Table 5 . The $p$-values $(p<0.05,0.01,0.001)$ were placed in superscript. 
Table 3. The content of studied elements $(\mathrm{Ca}, \mathrm{Cu}, \mathrm{Fe}, \mathrm{Mg})$ measured in rice and rice products.

\begin{tabular}{|c|c|c|c|c|c|c|c|c|c|c|c|c|c|}
\hline \multirow{2}{*}{$\begin{array}{l}\text { The Type of } \\
\text { the Rice and } \\
\text { Rice Product }\end{array}$} & \multirow[b]{2}{*}{$n$} & \multicolumn{3}{|c|}{$\mathrm{Ca}(\mathrm{mg} / \mathrm{kg})$} & \multicolumn{3}{|c|}{$\mathrm{Cu}(\mathrm{mg} / \mathrm{kg})$} & \multicolumn{3}{|c|}{$\mathrm{Fe}(\mathrm{mg} / \mathrm{kg})$} & \multicolumn{3}{|c|}{$\mathrm{Mg}(\mathrm{mg} / \mathrm{kg})$} \\
\hline & & $\begin{array}{c}\mathrm{X} \pm \mathrm{SD} \\
\text { (Min-Max) }\end{array}$ & $\begin{array}{c}\mathrm{Me} \\
\left(\mathrm{Q}_{1}-\mathrm{Q}_{3}\right) \\
\end{array}$ & $\begin{array}{l}\% \text { of RVI } \\
(800 \mathrm{mg}) \\
\end{array}$ & $\begin{array}{c}\mathrm{X} \pm \mathrm{SD} \\
\text { (Min-Max) }\end{array}$ & $\begin{array}{c}\mathrm{Me} \\
\left(\mathrm{Q}_{1}-\mathrm{Q}_{3}\right) \\
\end{array}$ & $\begin{array}{c}\text { \% of RVI } \\
\text { (1 mg) }\end{array}$ & $\begin{array}{c}\mathrm{X} \pm \mathrm{SD} \\
\text { (Min-Max) }\end{array}$ & $\begin{array}{c}\mathrm{Me} \\
\left(\mathrm{Q}_{1-} \mathrm{Q}_{3}\right) \\
\end{array}$ & $\begin{array}{c}\text { \% of RVI } \\
\text { (14 mg) }\end{array}$ & $\begin{array}{c}\mathrm{X} \pm \mathrm{SD} \\
\text { (Min-Max) }\end{array}$ & $\begin{array}{c}\mathrm{Me} \\
\left(\mathrm{Q}_{1}-\mathrm{Q}_{3}\right) \\
\end{array}$ & $\begin{array}{l}\text { \% of RVI } \\
(375 \mathrm{mg})\end{array}$ \\
\hline Basmati & 10 & $\begin{array}{c}193.5 \pm 61.9 \\
(133.3-343.9)\end{array}$ & $\begin{array}{c}181.9 \\
(151.8-208.3)\end{array}$ & 2 & $\begin{array}{l}2.4 \pm 0.4 \\
(1.9-3.1)\end{array}$ & $\begin{array}{c}2.2 \\
(2.0-2.6)\end{array}$ & 24 & $\begin{array}{c}6.5 \pm 7.9 \\
(2.4-28.9)\end{array}$ & $\begin{array}{c}4.0 \\
(3.4-5.1)\end{array}$ & 5 & $\begin{array}{c}379.0 \pm 378.6 \\
(143.0-1363.0)\end{array}$ & $\begin{array}{c}205.0 \\
(176.0-532.4)\end{array}$ & 10 \\
\hline Black & 6 & $\begin{array}{c}324.1 \pm 156.1 \\
(73.4-443.5)\end{array}$ & $\begin{array}{c}405.9 \\
(185.5-430.7)\end{array}$ & 4 & $\begin{array}{l}3.4 \pm 1.5 \\
(2.4-6.4)\end{array}$ & $\begin{array}{c}2.9 \\
(2.6-3.4)\end{array}$ & 34 & $\begin{array}{l}16.7 \pm 3.2 \\
(12.1-21.7)\end{array}$ & $\begin{array}{c}16.9 \\
(15.0-17.7)\end{array}$ & 12 & $\begin{array}{l}1167.8 \pm 280.5 \\
(997.1-1723.9)\end{array}$ & $\begin{array}{c}1034.3 \\
(1030.4-1186.9)\end{array}$ & 31 \\
\hline Brown & 10 & $\begin{array}{c}325.8 \pm 162.2 \\
(65.1-613.8)\end{array}$ & $\begin{array}{c}329.2 \\
(268.8-408.6)\end{array}$ & 4 & $\begin{array}{l}4.0 \pm 3.6 \\
(1.3-13.1)\end{array}$ & $\begin{array}{c}2.4 \\
(1.7-5.0)\end{array}$ & 40 & $\begin{array}{l}15.9 \pm 3.1 \\
(12.7-23.1)\end{array}$ & $\begin{array}{c}15.1 \\
(14.0-15.8)\end{array}$ & 11 & $\begin{array}{l}1017.0 \pm 386.5 \\
(149.3-1468.5)\end{array}$ & $\begin{array}{c}964.0 \\
(882.0-1351.5)\end{array}$ & 27 \\
\hline Parboiled & 10 & $\begin{array}{c}137.5 \pm 127.2 \\
(23.7-463.7)\end{array}$ & $\begin{array}{c}89.4 \\
(68.8-175.0)\end{array}$ & 2 & $\begin{array}{l}2.4 \pm 0.5 \\
(1.4-3.3)\end{array}$ & $\begin{array}{c}2.5 \\
(2.1-2.7)\end{array}$ & 24 & $\begin{array}{c}6.5 \pm 6.3 \\
(1.8-22.8)\end{array}$ & $\begin{array}{c}3.9 \\
(3.1-6.9)\end{array}$ & 5 & $\begin{array}{c}224.9 \pm 87.1 \\
(7.1-312.4)\end{array}$ & $\begin{array}{c}239.5 \\
(211.9-283.6)\end{array}$ & 6 \\
\hline Red & 5 & $\begin{array}{c}216.7 \pm 227.6 \\
(51.3-545.1)\end{array}$ & $\begin{array}{c}66.0 \\
(53.7-367.2)\end{array}$ & 2 & $\begin{array}{l}3.7 \pm 1.3 \\
(2.3-5.5)\end{array}$ & $\begin{array}{c}3.4 \\
(2.8-4.3)\end{array}$ & 37 & $\begin{array}{l}11.8 \pm 3.2 \\
(8.7-15.5)\end{array}$ & $\begin{array}{c}10.0 \\
(9.9-15.1)\end{array}$ & 9 & $\begin{array}{c}1241.7 \pm 305.6 \\
(1014.6-1754.3)\end{array}$ & $\begin{array}{c}1086.0 \\
(1061.5-1292.3)\end{array}$ & 33 \\
\hline Wild & 5 & $\begin{array}{c}294.6 \pm 143.5 \\
(83.7-421.2)\end{array}$ & $\begin{array}{c}367.2 \\
(209.6-391.5)\end{array}$ & 5 & $\begin{array}{c}12.6 \pm 2.4 \\
(10.5-16.6)\end{array}$ & $\begin{array}{c}11.7 \\
(11.5-12.8)\end{array}$ & 126 & $\begin{array}{c}17.9 \pm 3.1 \\
(14.0-21.8)\end{array}$ & $\begin{array}{c}17.7 \\
(16.1-20.0)\end{array}$ & 13 & $\begin{array}{l}1043.6 \pm 155.1 \\
(873.7-1292.3)\end{array}$ & $\begin{array}{c}1014.0 \\
(977.7-1060.0)\end{array}$ & 28 \\
\hline White & 11 & $\begin{array}{l}114.1 \pm 72.6 \\
(16.1-278.2)\end{array}$ & $\begin{array}{c}88.2 \\
(66.9-150.1)\end{array}$ & 1 & $\begin{array}{l}2.4 \pm 0.7 \\
(1.4-3.8)\end{array}$ & $\begin{array}{c}2.4 \\
(1.7-2.9)\end{array}$ & 24 & $\begin{array}{l}4.5 \pm 2.9 \\
(1.4-9.9)\end{array}$ & $\begin{array}{c}3.7 \\
(2.0-5.2)\end{array}$ & 3 & $\begin{array}{l}258.2 \pm 101.4 \\
(109.6-423.8)\end{array}$ & $\begin{array}{c}234.0 \\
(181.7-371.9)\end{array}$ & 7 \\
\hline Expanded & 8 & $\begin{array}{c}187.0 \pm 118.0 \\
(74.0-406.2)\end{array}$ & $\begin{array}{c}170.1 \\
(81.5-256.3)\end{array}$ & 2 & $\begin{array}{l}2.9 \pm 1.9 \\
(1.7-7.6)\end{array}$ & $\begin{array}{c}2.4 \\
(1.8-2.7)\end{array}$ & 29 & $\begin{array}{c}4.8 \pm 2.7 \\
(2.1-10.6)\end{array}$ & $\begin{array}{c}4.4 \\
(2.9-5.6)\end{array}$ & 3 & $\begin{array}{l}412.0 \pm 138.3 \\
(262.2-703.4)\end{array}$ & $\begin{array}{c}415.3 \\
(305.6-444.5)\end{array}$ & 11 \\
\hline Flakes & 12 & $\begin{array}{c}156.3 \pm 94.01 \\
(63.1-362.6)\end{array}$ & $\begin{array}{c}129.5 \\
(107.2-158.0)\end{array}$ & 2. & $\begin{array}{l}3.3 \pm 1.2 \\
(2.1-6.5)\end{array}$ & $\begin{array}{c}3.0 \\
(2.6-3.7)\end{array}$ & 33 & $\begin{array}{l}5.5 \pm 5.9 \\
(1.4-18.2)\end{array}$ & $\begin{array}{c}3.3 \\
(3.0-4.2)\end{array}$ & 4 & $\begin{array}{l}319.8 \pm 318.0 \\
(91.7-1086.2)\end{array}$ & $\begin{array}{c}200.1 \\
(137.4-305.7)\end{array}$ & 9 \\
\hline Flour & 6 & $\begin{array}{c}192.1 \pm 132.3 \\
(65.7-426.3)\end{array}$ & $\begin{array}{c}185.8 \\
(67.7-221.3)\end{array}$ & 2 & $\begin{array}{l}2.4 \pm 0.5 \\
(1.7-3.0)\end{array}$ & $\begin{array}{c}2.4 \\
(2.2-2.7)\end{array}$ & 24 & $\begin{array}{l}8.7 \pm 5.1 \\
(4.5-18.1)\end{array}$ & $\begin{array}{c}7.4 \\
(4.7-10.3)\end{array}$ & 6 & $\begin{array}{l}571.4 \pm 416.1 \\
(264.8-1385.1)\end{array}$ & $\begin{array}{c}433.6 \\
(333.4-578.2)\end{array}$ & 15 \\
\hline Pasta & 7 & $\begin{array}{c}276.8 \pm 279.5 \\
(73.5-867.0)\end{array}$ & $\begin{array}{c}185.6 \\
(85.6-355.2)\end{array}$ & 3 & $\begin{array}{l}2.4 \pm 0.7 \\
(1.4-3.2)\end{array}$ & $\begin{array}{c}2.8 \\
(1.7-2.9)\end{array}$ & 24 & $\begin{array}{l}3.9 \pm 1.5 \\
(2.1-6.3)\end{array}$ & $\begin{array}{c}3.5 \\
(2.4-5.6)\end{array}$ & 3 & $\begin{array}{l}125.7 \pm 59.5 \\
(81.6-250.4)\end{array}$ & $\begin{array}{c}107.7 \\
(86.9-146.7)\end{array}$ & 3 \\
\hline TOTAL & 99 & $\begin{array}{c}226.3 \pm 160.6 \\
(16.1-867.0)\end{array}$ & $\begin{array}{c}176.1 \\
(85.6-362.6)\end{array}$ & - & $\begin{array}{l}3.6 \pm 2.8 \\
(1.3-16.6)\end{array}$ & $\begin{array}{c}2.7 \\
(2.1-3.4)\end{array}$ & - & $\begin{array}{c}9.4 \pm 7.0 \\
(1.4-28.8)\end{array}$ & $\begin{array}{c}5.9 \\
(3.4-15.1)\end{array}$ & - & $\begin{array}{c}618.0 \pm 498.4 \\
(7.1-1811.5)\end{array}$ & $\begin{array}{c}382.3 \\
(211.9-1030.4)\end{array}$ & - \\
\hline
\end{tabular}

X—mean, SD—standard deviation, Min—minimum, Max—maximum, Me—median, $\mathrm{Q}_{1}$-lower quartile, $\mathrm{Q}_{3}$ —upper quartile, RVI—reference value intake, calculated per $100 \mathrm{~g}$ of product. 
Table 4. The content of $\mathrm{Mn}$, Se and $\mathrm{Zn}$ in rice and rice products.

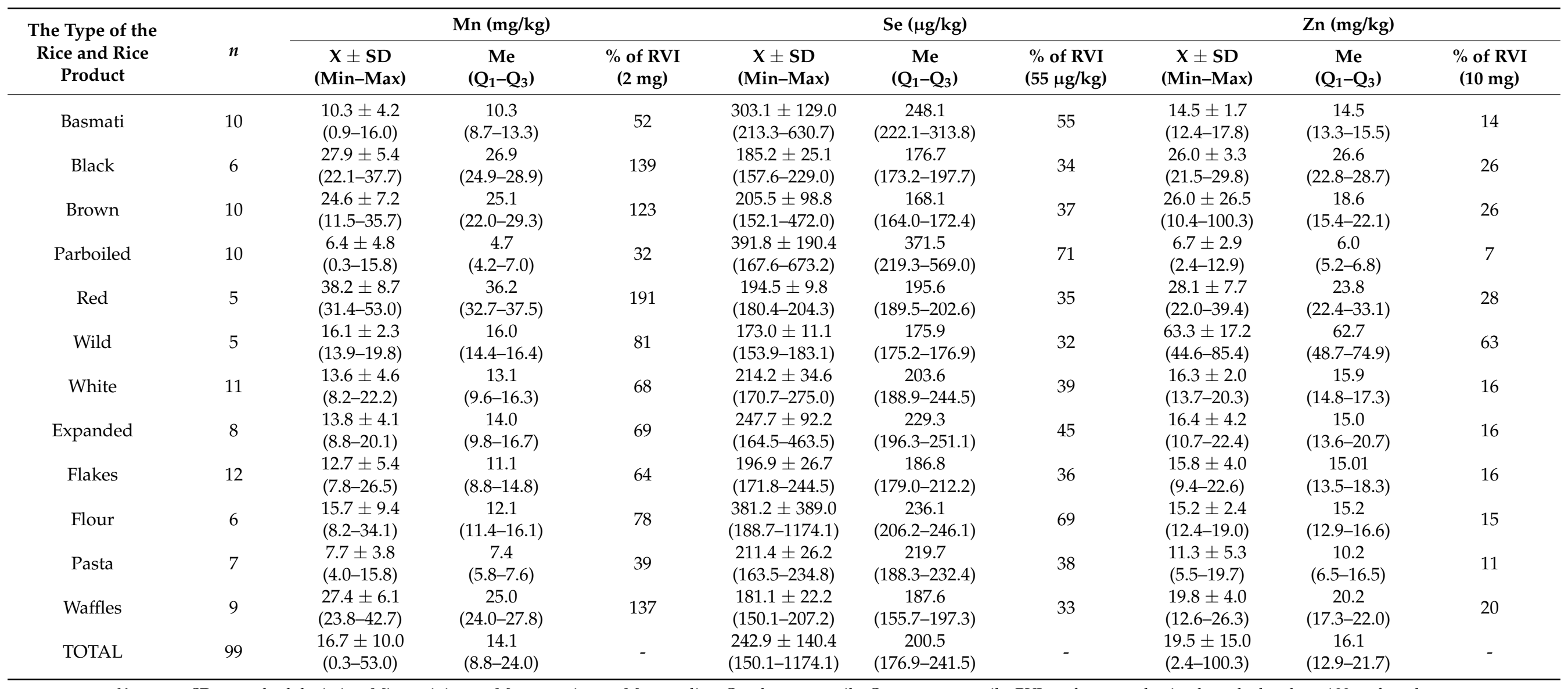

$\mathrm{X}-$ mean, SD—standard deviation, Min—minimum, Max—maximum, Me—median, $\mathrm{Q}_{1}$-lower quartile, $\mathrm{Q}_{3}$-upper quartile, RVI—reference value intake, calculated per $100 \mathrm{~g}$ of product. 
Table 5. The statistically relevant differences in the content of the studied elements considering the type of product.

\begin{tabular}{|c|c|c|c|c|c|c|c|c|}
\hline & Basmati & Black & Brown & Parboiled & Wild & Flakes & Pasta & Waffles \\
\hline Black & & & & $\begin{array}{l}\mathrm{Mn}^{0.001} \\
\mathrm{Zn}^{0.001}\end{array}$ & & & & \\
\hline Brown & $\mathrm{Se}^{0.01}$ & & & $\begin{array}{c}\mathrm{Mn}^{0.001} \\
\mathrm{Se}^{0.05} \\
\mathrm{Zn}^{0.01}\end{array}$ & & $\mathrm{Fe}^{0.01}$ & $\begin{array}{c}\mathrm{Fe}^{0.05} \\
\mathrm{Mg}^{0.01} \\
\mathrm{Mn}^{0.01}\end{array}$ & \\
\hline Red & $\mathrm{Mn}^{0.01}$ & & & $\begin{array}{c}\mathrm{Mn}^{0.001} \\
\mathrm{Zn}^{0.05}\end{array}$ & & $\begin{array}{l}\mathrm{Mg}^{0.05} \\
\mathrm{Mn}^{0.05}\end{array}$ & $\begin{array}{l}\mathrm{Mg}^{0.001} \\
\mathrm{Mn}^{0.001} \\
\mathrm{Zn}^{0.001}\end{array}$ & \\
\hline Wild & $\begin{array}{l}\mathrm{Se}^{0.05} \\
\mathrm{Zn}^{0.05}\end{array}$ & & & $\mathrm{Zn}^{0.001}$ & & $\mathrm{Fe}^{0.05}$ & $\begin{array}{c}\mathrm{Mg}^{0.01} \\
\mathrm{Zn}^{0.01}\end{array}$ & \\
\hline White & & $\mathrm{Fe}^{0.05}$ & $\mathrm{Fe}^{0.05}$ & $\mathrm{Se}^{0.05}$ & $\mathrm{Fe}^{0.05}$ & & & $\begin{array}{c}\mathrm{Ca}^{0.01} \\
\mathrm{Fe}^{0.01} \\
\mathrm{Mg}^{0.01}\end{array}$ \\
\hline Flakes & & $\mathrm{Mg}^{0.05}$ & & & & & & $\begin{array}{c}\mathrm{Ca}^{0.05} \\
\mathrm{Fe}^{0.01} \\
\mathrm{Mg}^{0.01}\end{array}$ \\
\hline Pasta & & $\begin{array}{l}\mathrm{Mg}^{0.001} \\
\mathrm{Mn}^{0.01} \\
\mathrm{Zn}^{0.001}\end{array}$ & & & & & & $\begin{array}{c}\mathrm{Fe}^{0.05} \\
\mathrm{Mg}^{0.001} \\
\mathrm{Mn}^{0.001}\end{array}$ \\
\hline Waffles & $\begin{array}{c}\mathrm{Mg}^{0.001} \\
\mathrm{Se}^{0.05}\end{array}$ & & & $\begin{array}{c}\mathrm{Ca}^{0.05} \\
\mathrm{Mg}^{0.001} \\
\mathrm{Se}^{0.05} \\
\mathrm{Zn}^{0.001}\end{array}$ & & & & \\
\hline
\end{tabular}

\section{Discussion}

In this investigation, we assessed the quality of 12 different groups of rice products available on the Polish market by determining the content of macro- and microelements. The results obtained by other authors are presented in Table 6 . 
Table 6. Content of elements in rice and rice products determined by other authors.

\begin{tabular}{|c|c|c|c|c|c|c|c|c|}
\hline \multirow{2}{*}{$\begin{array}{l}\text { Type of Product } \\
\text { [Ref.] }\end{array}$} & \multirow{2}{*}{$n$} & \multicolumn{7}{|c|}{ Element } \\
\hline & & $\mathrm{Ca}(\mathrm{mg} / \mathrm{kg})$ & $\mathrm{Cu}(\mathrm{mg} / \mathrm{kg})$ & $\mathrm{Fe}(\mathrm{mg} / \mathrm{kg})$ & $\mathrm{Mg}(\mathrm{mg} / \mathrm{kg})$ & $\mathrm{Mn}(\mathrm{mg} / \mathrm{kg})$ & Se $(\mu \mathrm{g} / \mathrm{kg})$ & $\mathrm{Zn}(\mathrm{mg} / \mathrm{kg})$ \\
\hline \multicolumn{9}{|l|}{ Brown Rice } \\
\hline$[16]$ & 9 & Min-Max: 87.1-114 & Min-Max: 1.3-4.6 & Min-Max: 13.3-14.5 & Min-Max: 160-1630 & Min-Max: 7.6-11.8 & - & - \\
\hline [17] & 6 & - & $X=3.1$ & $\mathrm{X}=11.5$ & & $X=28.9$ & $X=104$ & $X=22.5$ \\
\hline [18] & 11 & $\mathrm{X}=64 \pm 9$ & $X=1.6 \pm 0.4$ & $X=14.0 \pm 2.1$ & $X=1064 \pm 87$ & $X=21.5 \pm 4.4$ & $X=30 \pm 20$ & $X=15.9 \pm 2.3$ \\
\hline [19] & 16 & $X=104 \pm 37.9$ & $X=3.0 \pm 1.1$ & $X=20.1 \pm 7.8$ & $X=1205 \pm 335$ & $X=26.5 \pm 12.2$ & $X=131 \pm 57$ & $X=20.2 \pm 2.73$ \\
\hline \multirow[t]{2}{*}{ [20] } & 33 & $X=72.6 \pm 32.6$ & $X=5.5 \pm 5.4$ & $X=32 \pm 26.1$ & $X=1140 \pm 214$ & $X=20.1 \pm 10.5$ & $X=41 \pm 57$ & $X=18.8 \pm 4.3$ \\
\hline & 51 & $\begin{array}{l}(35.6-211.5) \\
-\end{array}$ & $\begin{array}{c}\text { (Min-Max: 2.2-29.1) } \\
\text { X = 2.35 }\end{array}$ & $\begin{array}{c}\text { (Min-Max: 12.1-119.1) } \\
\text { X }=18.6\end{array}$ & (Min-Max: 761-1550) & $\begin{array}{c}\text { (Min-Max: 12.4-74.4) } \\
X=15.5\end{array}$ & (Min-Max: 5-300) & (Min-Max: 13.9-34) \\
\hline \multirow[b]{2}{*}{ [22] } & 51 & - & $\begin{array}{c}x=2.35 \\
\text { (Min-Max: 1.4-3.9) }\end{array}$ & $\begin{array}{c}\lambda=18.6 \\
\text { (Min-Max: } 10.0-65.2)\end{array}$ & & $\begin{array}{c}X=15.5 \\
\text { (Min-Max: 8.2-24.2) }\end{array}$ & - & $\begin{array}{c}X=21.0 \\
\text { (Min-Max: 9.0-29.4) }\end{array}$ \\
\hline & 51 & - & $X=4.4$ & - & - & $\begin{array}{l}X=20 \\
\end{array}$ & $\begin{array}{c}X=39 \\
\end{array}$ & $\begin{array}{l}X=28 \\
\end{array}$ \\
\hline Our results & 10 & $\begin{array}{c}X=325.8 \pm 162.2 \\
\text { (Min-Max: } 65.1-613.8 \text { ) }\end{array}$ & $\begin{array}{c}\text { (Min-Max: 0.9-6.5) } \\
\quad \text { X=4.0 } 3.6 \\
\text { (Min-Max: 1.3-13.1) }\end{array}$ & $\begin{array}{c}X=15.9 \pm 3.1 \\
\text { (Min-Max: } 12.7-23.1 \text { ) }\end{array}$ & $\begin{array}{c}X=1017.0 \pm 386.5 \\
\text { (Min-Max: } 149.3-1468.5)\end{array}$ & $\begin{array}{c}\text { (Min-Max: 10-34) } \\
\text { X }=24.6 \pm 7.2 \\
\text { (Min-Max: } 11.5-35.7)\end{array}$ & $\begin{array}{c}\text { (Min-Max: 15-80) } \\
\text { X }=205.5 \pm 98.8 \\
\text { (Min-Max: 152.1-472.0) }\end{array}$ & $\begin{array}{c}\text { (Min-Max: 20-36) } \\
\quad \text { X }=26.0 \pm 26.5 \\
\text { (Min-Max: } 10.4-100.3 \text { ) }\end{array}$ \\
\hline \multicolumn{9}{|l|}{ White Rice } \\
\hline \multirow{2}{*}{$\begin{array}{l}{[17]} \\
{[18]}\end{array}$} & 5 & - & $X=2.3$ & $X=3.7$ & - & $X=7.8$ & $X=92$ & $X=13.1$ \\
\hline & 56 & $X=32 \pm 18$ & $X=1.8 \pm 0.6$ & $X=6.8 \pm 1.5$ & $X=225 \pm 63$ & $X=1.8 \pm 0.6$ & $X=200 \pm 190$ & $X=13.5 \pm 3.4$ \\
\hline [19] & 9 & $\begin{array}{l}X=127 \pm 141 \\
X=37.7 \pm 9.1\end{array}$ & $X=1.7 \pm 0.6$ & $X=22.3 \pm 37.9$ & $X=371 \pm 127$ & $X=10.5 \pm 3.7$ & $X=108 \pm 66$ & $X=15.6 \pm 1.9$ \\
\hline \multirow{2}{*}{ [20] } & 21 & $\begin{array}{c}\text { (Min-Max: 18.6-63) } \\
X=1141+72.6\end{array}$ & $X=3.1 \pm 2.0$ & $\begin{array}{c}X=7.9 \pm 2.9 \\
\end{array}$ & $X=259 \pm 44$ & $X=9.4 \pm 1.7$ & $X=40 \pm 35$ & $X=14.7 \pm 1.8$ \\
\hline & 11 & $\begin{array}{c}\mathrm{X}=114.1 \pm 72.6 \\
\text { (Min-Max: } 16.1-278.2)\end{array}$ & (Min-Max: 0.9-8.1) & $\begin{array}{c}\text { (Min-Max: 3.6-17.3) } \\
X=4.5 \pm 2.9\end{array}$ & $\begin{array}{c}\text { (Min-Max: 191-341) } \\
\text { X=258.2 } \pm 101.4\end{array}$ & $\begin{array}{c}\text { (Min-Max: 7.0-12.7) } \\
X=13.6 \pm 4.6\end{array}$ & $\begin{array}{c}\text { (Min-Max: 13-137) } \\
X=214.2 \pm 34.6\end{array}$ & $\begin{array}{l}\text { (Min-Max: 12-17.7) } \\
x=163+20\end{array}$ \\
\hline Our results & 10 & (Min-Max: 16.1-278.2) & $\begin{array}{c}X=2.4 \pm 0.7 \\
\text { (Min-Max: } 1.4-3.8)\end{array}$ & $\begin{array}{c}X=4.5 \pm 2.9 \\
\text { (Min-Max: } 1.4-9.9)\end{array}$ & $\begin{array}{c}X=258.2 \pm 101.4 \\
\text { (Min-Max: } 109.6-423.8)\end{array}$ & $\begin{array}{c}X=13.6 \pm 4.6 \\
\text { (Min-Max: 8.2-22.2) }\end{array}$ & $\begin{array}{c}X=214.2 \pm 34.6 \\
\text { (Min-Max: } 170.7-275.0)\end{array}$ & $\begin{array}{c}X=16.3 \pm 2.0 \\
\text { (Min-Max: } 13.7-20.3)\end{array}$ \\
\hline \multicolumn{9}{|l|}{ Parboiled Rice } \\
\hline \multirow{2}{*}{ Our results } & 10 & $X=137.5 \pm 127.2$ & $X=2.4 \pm 0.5$ & $X=6.5 \pm 6.3$ & $\mathrm{X}=224.9 \pm 87.1$ & $\mathrm{X}=6.4 \pm 4.8$ & $X=391.8 \pm 190.4$ & $X=6.7 \pm 2.9$ \\
\hline & & (Min-Max: 23.7-463.7) & (Min-Max: 1.4-3.3) & (Min-Max: 1.8-22.8) & (Min-Max: 7.1-312.4) & (Min-Max: 0.3-15.8) & (Min-Max: 167.6-673.2) & (Min-Max: 2.4-12.9) \\
\hline \multicolumn{9}{|l|}{ Wild Rice } \\
\hline \multirow{3}{*}{$\begin{array}{c}{[18]} \\
\text { Our results }\end{array}$} & 6 & $\mathrm{X}=238 \pm 170$ & $X=3.3 \pm 1.9$ & $\mathrm{X}=7.8 \pm 1.2$ & $X=561 \pm 170$ & $X=5.5 \pm 0.8$ & $\mathrm{X}=120 \pm 40$ & $X=24.7 \pm 4.6$ \\
\hline & 5 & $X=294.6 \pm 143.5$ & $\mathrm{X}=12.6 \pm 2.4$ & $X=17.9 \pm 3.1$ & $X=1043.6 \pm 155.1$ & $X=16.1 \pm 2.3$ & $X=173.0 \pm 11.1$ & $X=63.3 \pm 17.2$ \\
\hline & & (Min-Max: 83.7-421.2) & (Min-Max: 10.5-16.6) & (Min-Max: 14.0-21.8) & (Min-Max: 873.7-1292.3) & (Min-Max: 13.9-19.8) & (Min-Max: 153.9-183.1) & (Min-Max: 44.6-85.4) \\
\hline \multicolumn{9}{|l|}{ Rice } \\
\hline \multirow{3}{*}{$\begin{array}{l}{[23]} \\
{[24]}\end{array}$} & 9* & $X=84.5$ & $X=1.1$ & $X=20.7$ & - & - & - & $X=23.4$ \\
\hline & 56 & & $X=3.3 \pm 0.9$ & $X=39.4 \pm 17.6$ & - & $X=4.4 \pm 1.4$ & $X=280 \pm 200$ & $\mathrm{X}=9.8 \pm 2.8$ \\
\hline & & & (min-Max:0.7-6.9) & (Min-Max: 11.8-90.6) & - & (Min-Max: 2.3-8.4) & (Min-Max: 0-630) & (Min-Max: 6.3-20.3) \\
\hline [25] & 23 & $X=71.5 \pm 7.3$ & $X=3.7 \pm 0.2$ & - & - & $\mathrm{X}=8.5 \pm 0.5$ & $X=401 \pm 92$ & $X=10.2 \pm 0.3$ \\
\hline \multirow{3}{*}{\multicolumn{2}{|c|}{$\begin{array}{l}\text { Our results } \\
\text { (rice in total) }\end{array}$}} & - & $X=2.0 \pm 0.5$ & - & & $X=4.7 \pm 0.6$ & $X=25.6 \pm 10$ & $X=13.2 \pm 2.1$ \\
\hline & & $X=226.3 \pm 160.6$ & $X=3.6 \pm 2.8$ & $X=9.4 \pm 7.0$ & $X=618.0 \pm 498.4$ & $X=16.7 \pm 10.0$ & $X=242.9 \pm 140.4$ & $X=19.5 \pm 15.0$ \\
\hline & & (Min-Max: 16.1-867.0) & (Min-Max: 1.3-16.6) & (Min-Max: 1.4-28.8) & (Min-Max: 7.1-1811.5) & (Min-Max: 0.3-53.0) & (Min-Max: 150.1-1174.1) & (Min-Max: 2.4-100.3) \\
\hline \multicolumn{9}{|l|}{ Rice Flour } \\
\hline \multirow{3}{*}{$\begin{array}{c}{[17]} \\
\text { Our results }\end{array}$} & 4 & & $X=2.4$ & $X=31.2$ & & $X=22.5$ & $X=117$ & $\mathrm{X}=14.9$ \\
\hline & 6 & $X=192.1 \pm 132.3$ & $\mathrm{X}=2.4 \pm 0.5$ & $\mathrm{X}=8.7 \pm 5.1$ & $X=571.4 \pm 416.1$ & $X=15.7 \pm 9.4$ & $\mathrm{X}=381.2 \pm 389.0$ & $X=15.2 \pm 2.4$ \\
\hline & & (Min-Max: 65.7-426.3) & (Min-Max: 1.7-3.0) & (Min-Max: 4.5-18.1) & (Min-Max: 264.8-1385.1) & (Min-Max: 8.2-34.1) & (Min-Max: 188.7-1174.1) & (Min-Max: 12.4-19.0) \\
\hline
\end{tabular}


Compared to other plant products considered to be sources of $\mathrm{Ca}$ (such as legumes), the Ca content in our study material was generally low; however, most of the other authors $[16,19,20,23,25]$ observed even lower Ca levels than that measured in our samples ( Tables 3 and 6). Only Pinto et al. determined in parboiled rice a higher Ca level than that in this research [18]. The parboiling process increases the Ca content due to specific conditions, such as soaking time and proper temperature [27]. The tested products could not be regarded as a source of $\mathrm{Ca}$ in the diet of European adults (Table 3). Insufficient $\mathrm{Ca}$ intake is still a worldwide problem. Taking into account $\mathrm{Ca}$ intake with a whole day diet, most of the available research determines the insufficient intake of this nutrient [5,28]. In the study by Grygorieva et al., among Ukrainian adults, only $7.8 \%$ of subjects consumed more than $1200 \mathrm{mg}$ of Ca per day [29]. The problem of inadequate Ca consumption amid Spanish adolescents and elderly people population was observed as well [30]. Considering the daily intake levels, Kashian and Fathivand observed that rice consumption fulfilled the recommended dietary allowances (RDA) norm within $1 \%$ of the Iranian population [23]. Similar observations were found in the study conducted by Pinto et al. among the Iberian (Portugal and Spain) population; daily Ca requirements by rice consumption were met from $0.2 \%$ to $1.3 \%$ of the RDA norm [18]. Other Spanish researchers also reported that daily Ca intake through rice consumption ranged from $0.6 \%$ of RDA norm for white rice to $1.07 \%$ of RDA for brown rice. However, when brown rice was replaced by white rice, the daily Ca intake was reduced by $47 \%$ [16].

The $\mathrm{Cu}$ levels measured in this investigation are in agreement with those reported by other authors [16-22,24-26]. Taking into account the recommendations of $\mathrm{Cu}$ intake, rice products available on the Polish market could be regarded as a source of this element in the daily diet of European adults (Table 3). Regarding rice consumption, observations were made by Antoine et al. that among the Jamaican population, polished rice intake fulfilled daily $\mathrm{Cu}$ requirements in 13\%, while unprocessed grains in 23\% [19]. Among Malawian adults, daily rice intake covered $19 \%$ of the RDA, while amid the Nigerian population, $31 \%[20,25]$. Lower daily $\mathrm{Cu}$ intake was reported for Iberian populations and ranged from $6.4 \%$ to $13 \%$ of the RDA norm [18]. Jo and Todorov demonstrated that in rice grains, $\mathrm{Cu}$ is concentrated mainly in the bran and outer layers of the endosperm. When brown rice was polished to white rice (removal $18 \%$ of grain weight), the $\mathrm{Cu}$ content decreased by nearly $20 \%$ from $2.29 \mathrm{mg} / \mathrm{kg}$ to $1.91 \mathrm{mg} / \mathrm{kg}$ [31]. Moreover, Hensawang et al. reported that extra thorough rice polishing reduced $\mathrm{Cu}$ content by 35\% [32]. Ortiz and Camara-Martos determined $\mathrm{Cu}$ bioaccessibility through solubility and dialyzability assays among eight of the most consumed rice varieties in Argentina and Spain. The bioaccessibility ranged from $24 \%$ to $80 \%$, while dialyzability ranged from $4 \%$ to $41 \%$ [33]. In the study by Babaali et al., among the Iranian population, the average daily $\mathrm{Cu}$ intake exceeded the estimated average requirements (EAR) and was determined at $3.8 \mathrm{mg}$; fresh fruit and vegetables as the main sources $(31 \%)$ of this element were indicated [34]. More than three times lower dietary $\mathrm{Cu}$ consumption (1.2 $\mathrm{mg} /$ day) was reported in the investigation conducted among Japanese adults. The authors observed that white rice accounted for approximately $20-30 \%$ of the total intake of this element [35].

Considering the Fe content in rice samples, some other authors [16-18] reported comparable results to ours (Tables 3 and 6). However, higher Fe levels were also frequently reported $[19-21,23,24]$. None of the subgroups tested can be a source of Fe for the adult European population (Table 3). Women of childbearing age are at risk of inadequate Fe intake due to high body requirements for this element. Considering Fe intake with whole day diet among Spanish adults, only $17 \%$ of women had sufficient intake, while among men, it was $57.3 \%$ of participants [36]. Similar observations were reported among Polish adolescents; females had significantly lower Fe intake than males [37]. On the other hand, both white and brown rice fulfilled daily RDA for men in Jamaica at 20\% and $18 \%$, while for women it was $8.8 \%$ and $8 \%$, respectively [19]. Among the Iranian population, rice consumption covered the recommended daily intake in $14 \%$ for women and $31 \%$ for men [23]. Fe in rice grains is unevenly distributed and located mainly in the 
pericarp and aleurone layers. Five times higher Fe concentration was observed in the dorsal compared to the ventral side of the bran layer. The polishing process $(18 \%$ removal of grain weight) reduced Fe content in brown rice from $10.4 \mathrm{mg} / \mathrm{kg}$ to $3.6 \mathrm{mg} / \mathrm{kg}$ in white rice; therefore $66 \%$ of Fe was removed [31]. Besides the total content of Fe in rice, the presence of compounds negatively affecting its bioavailability (such as phytic acid, polyphenols, tannins and dietary fiber) should be taken into account. Average Fe solubility was within the range of $80 \%$ in rice grains, except for brown rice. The lowest bioaccessible percentage, despite the high Fe content, was a result of a high concentration of the antinutritional factors mentioned above [33].

The Mg levels determined in our research (Table 3) were close to those reported in other investigations [16,18,20,24]. Except for six groups of products (basmati, expanded, parboiled, white rice, flakes and pasta), the remaining rice products can be a source of $\mathrm{Mg}$ for European adults (Table 3). Taking into account the daily rice consumption, Portuguese and Spanish citizens fulfilled the RDA norm from $2.4 \%$ to $11.8 \%$ among women and between $1.8 \%$ and $9 \%$ among men. Amid the Jamaican population, white rice consumption covered $\mathrm{Mg}$ daily requirements in $8.2 \%$ in females and $6.3 \%$ in males, while brown rice in $27 \%$ and $20 \%$, respectively [19]. Cano-Lamarid et al. observed that the replacement of brown rice with white rice in the diet may result in a reduction in daily $\mathrm{Mg}$ intake by 70\% [16]. Daily Mg intake was generally adequate in research conducted by SchiefermeierMach et al.; however, in nearly one-third of participants, the coverage of requirements was not met [38]. Similar results were observed among the Iranian population-22.4\% of the study group had an $\mathrm{Mg}$ intake lower than the EAR norm [34].

A lower Mn content than found in this investigation was reported by Pinto et al. in the case of white and wild rice, and by Halder et al. also, in white rice [18,24], whereas most of the other researchers determined similar levels of Mn in rice samples [16,17,19-22,25,26]. Every subgroup of the rice products tested can be a rich source of $\mathrm{Mn}$ in the diet of the adult European population (Table 4). One portion (300 g) of red rice reached the TUIL of $\mathrm{Mn}$ in our investigation. Moreover, when another foodstuff consumed daily is added, there is a potential risk of elevated Mn levels in the body, which could pose negative effects. The investigation by Choi and Bae among Korean adults demonstrated that Mn intake with diet covered the adequate intake (AI) norm in 103\% for men and $110 \%$ for women and the main sources of this element were cereal and cereal products [39]. Regarding the daily intake, in Antoine's et al. investigation, white rice consumption fulfilled the recommended level of intake for women at $41 \%$ and men at $32 \%$, while brown rice at $105 \%$ and $82 \%$, respectively [19]. Pinto et al. estimated that the AI norm for the Iberian females through rice consumption was covered from $10.8 \%$ to $42.5 \%$ and from $8.5 \%$ to $33.3 \%$ for Iberian males [18]. The total content of $\mathrm{Mn}$ in the investigation conducted by Ortiz and Camara-Martoz ranged from $2.5 \mathrm{mg} / \mathrm{kg}$ to $14 \mathrm{mg} / \mathrm{kg}$. Taking into account the bioaccessibility, the concentration of soluble Mn varied from $0.35 \mathrm{mg} / \mathrm{kg}$ to $2.52 \mathrm{mg} / \mathrm{kg}$, while the dialyzable fluctuated from $0.15 \mathrm{mg} / \mathrm{kg}$ to $0.64 \mathrm{mg} / \mathrm{kg}$. Moreover, the authors observed a statistically significant negative interaction between the content of soluble Mn and fiber in the studied grains [33]. Hensawang et al. found that the polishing process decreased the Mn concentration by $62 \%$ [32]. The content in unpolished and polished grains differed significantly $(p<0.01, \mathrm{r}=-0.747)$. On the other hand, Jo and Todorov reported lower Mn reduction (43\%) during the polishing process. The Mn in rice grain, similar to $\mathrm{Fe}$, is localized mainly in the pericarp and aleurone layers; however, this trace element is also present in the endosperm. In the dorsal, compared to the ventral, side of the bran layer, a five times higher Mn concentration was observed [31].

Considering Se levels in rice products, our results were higher than reported by most of the other researchers [17-20,22,26]. Only Halder et al. measured a fairly similar content, while Aderide et al. determined greater amounts of Se in tested rice [24,25]. Due to considerably high Se levels, every subgroup of the rice products tested can be regarded as a source of this trace element for European adults (Table 4). Cereals and meat were indicated as the main sources of Se in the diet of Italian adults. The daily level of Se consumption was 
sufficient; for women, it was estimated at $65 \mu \mathrm{g} /$ day and for men at $67 \mu \mathrm{g} /$ day [40]. Similar results were observed among Belgian adults [41]. Taking into account rice consumption, among the Brazilian population, rice intake covered $5 \%$ of the daily Se requirement [25]. For Jamaican adults, brown rice consumption covered $17 \%$ of the daily norm, while white rice covered $14 \%$ [19]. In the research conducted by Jo and Todorov, the polishing process slightly decreased Se content in whole grain rice from $150 \mu \mathrm{g} / \mathrm{kg}$ to $144 \mu \mathrm{g} / \mathrm{kg}$ in white rice. Additional analysis showed that Se is evenly distributed through the grain [31].

The $\mathrm{Zn}$ content in this study (Table 4) was comparable to the results obtained by most of the other authors cited in Table 6 [17-21,23]. In three studies, the measured Zn levels were lower than observed in our samples [24-26]. Only Rothenberg et al. reported higher Zn levels [22]. Except for basmati and parboiled rice as well as pasta, the remaining studied products could be a source of $\mathrm{Zn}$ for the adult European population (Table 4). In the investigation of Pinto et. al, consumption of rice fulfilled the RDA norm by $1.8 \%$ to $10.9 \%$ [18]. Fairly similar fulfilment of the RDA norm (9\%) was observed amid Nigerian women [25]. During the polishing process, the content of $\mathrm{Zn}$ was reduced by $23 \%$ (from $18.3 \mathrm{mg} / \mathrm{kg}$ to $14.1 \mathrm{mg} / \mathrm{kg}$ ). Generally, $\mathrm{Zn}$ is distributed homogeneously through the grain; however, the bran layer contains higher average Zn levels (20-80 mg/ $\mathrm{kg}$ ) compared to the endosperm $(13 \mathrm{mg} / \mathrm{kg})$ [31]. The high content of antinutritional components in bran impaired Zn bioaccessibility from rice grains. In the study by Ortiz and Camara-Martoz, the total $\mathrm{Zn}$ level ranged from $8.8 \mathrm{mg} / \mathrm{kg}$ to $13 \mathrm{mg} / \mathrm{kg}$ and the following soluble and dialyzable contents were reported: $0.9-3.3 \mathrm{mg} / \mathrm{kg}$ and $0.2-1.3 \mathrm{mg} / \mathrm{kg}$, respectively. Thus, $\mathrm{Zn}$ bioaccessibility was quite low-the solubility did not exceed $15 \%$-while dialyzability was lower than $3 \%$. It was observed that vegetable proteins were negatively correlated $(p<0.05$, $\mathrm{r}=-0.409$ ) with Zn bioaccessibility [33]. Considering the overall daily diet, among Chinese adults, over one-third are at risk of $Z n$ deficiency due to intake below the EAR norm. Grain consumption contributed to nearly $40 \%$ of total dietary Zn intake [42]. On the other hand, among American adults, daily Zn intake above RDA norm was reported [43].

To the best of our knowledge, there are limited data regarding the TUIL for elements consumed with diet. The risk of $\mathrm{Ca}$ intake with food products reaching TUIL is very low [44]. In a large European cohort study, exceedances of TUIL considering Fe (among women and men) and $\mathrm{Mg}$ (among men) were reported. The risk of over intake was observed among those who consumed dietary supplements [45]. No nutrient intakes with a diet above the TUIL were reported among the Canadian population. However, study participants who took dietary supplements were at risk of overly high $\mathrm{Mg}$ and $\mathrm{Zn}$ intake [46]. In turn, our analyses showed that there could be a potential risk of over intake of Mn through the whole day diet (even without supplementation). Therefore, we see a great need to conduct research focused on this topic.

\section{Conclusions}

Taking all of the above described into account, our study revealed that all of the studied subgroups of rice products available on the Polish market can be regarded as a source of $\mathrm{Cu}, \mathrm{Mn}$, and Se, while most of the products can be a source of $\mathrm{Mg}$ and $\mathrm{Zn}$ in the diet of the adult European population. Therefore, this fact should be highlighted in nutritional education. Considering the tolerable upper intake level of the studied elements, the tested products could be regarded as safe to consume. However, we see a great need to assess the daily intake of $\mathrm{Mn}$ with all products consumed in the diet due to the possible risk of excessive intake.

Author Contributions: Conceptualization, J.B. and R.M.-Ż.; methodology, K.S. and J.S; software, A.P.J. and P.N.; validation, J.B., M.G. and K.S.; formal analysis, A.M.; investigation, J.B., P.N., R.M.-Ż. and J.S.; resources, J.B. and P.N.; data curation, K.S. and R.M.-Ż.; writing-original draft preparation, J.B. and R.M.-Ż.; writing-review and editing, J.B. and P.N.; visualization, J.B. and R.M.-Ż.; supervision, K.S and J.B.; project administration, J.B., R.M.-Ż. and K.S.; funding acquisition, J.B. All authors have read and agreed to the published version of the manuscript. 
Funding: This research was supported by the Medical University of Białystok (SUB/3/DN/21/001/2216).

Institutional Review Board Statement: Not applicable.

Informed Consent Statement: Not applicable.

Data Availability Statement: The data presented in this study is available on request from the corresponding author.

Conflicts of Interest: The authors declare no conflict of interest.

\section{References}

1. OECD-FAO Agricultural Outlook (Edition 2020), OECD Agriculture Statistics (Database). Available online: https:/ /doi.org/10.1 $787 / 4919645 f-e n$ (accessed on 25 May 2021).

2. Arendt, E.K.; Zannini, E. Cereal Grains for the Food and Beverage Industries (Woodhead Publishing Series in Food Science, Technology and Nutrition); Woodhead Publishing: Cambridge, UK, 2013; pp. 113-154.

3. Juliano, B.O. Rice Chemistry and Quality; Island Publishing House: Science City of Muñoz, Philippines, 2003; pp. $25-49$.

4. Ito, V.C.; Lacerda, L.G. Black rice (Oryza sativa L.): A review of its historical aspects, chemical composition, nutritional and functional properties, and applications and processing technologies. Food Chem. 2019, 301, 125304. [CrossRef]

5. Koch, W.; Czop, M.; Nawrocka, A.; Wiacek, D. Contribution of major groups of food products to the daily intake of selected elements—results from analytical determinations supported by chemometric analysis. Nutrients 2020, 12, 3412. [CrossRef] [PubMed]

6. Głąbska, D.; Włodarek, D.; Kołota, A.; Czekajło, A.; Drozdzowska, B.; Pluskiewicz, W. Assessment of mineral intake in the diets of Polish postmenopausal women in relation to their BMI-the RAC-OST-POL study: Mineral intake in relation to BMI. J. Health Popul. Nutr. 2016, 35, 23. [CrossRef]

7. Pravina, P.; Sayaji, D.; Avinash, M. Calcium and its role in human body. Int. J. Res. Pharm. Biomed. Sci. 2013, 4, 659-668.

8. Scheiber, I.; Dringen, R.; Mercer, J.F.B. Interrelations between essential metal ions and human diseases. In Copper: Effects of Deficiency and Overload; Sigel, A., Sigel, H., Sigel, R.K.O., Eds.; Springer: Dordrecht, The Netherlands, 2013; pp. 359-387.

9. Abbaspour, N.; Hurrell, R.; Kelishadi, R. Review on iron and its importance for human health. J. Res. Med. Sci. 2014, 19, 164-174. [PubMed]

10. Gröber, U.; Schmidt, J.; Kisters, K. Magnesium in prevention and therapy. Nutrients 2015, 7, 8199-8226. [CrossRef]

11. Avila, D.S.; Puntel, R.L.; Aschner, M. Metal Ions in Life Sciences. In Interrelations between Essential Metal Ions and Human Diseases; Sigel, A., Sigel, H., Sigel, R.K.O., Eds.; Springer: Dordrecht, The Netherlands, 2013; pp. 199-227.

12. Kieliszek, M.; Błażejak, S. Current knowledge on the importance of selenium in food for living organisms: A review. Molecules 2016, 21, 609. [CrossRef]

13. Roohani, N.; Hurrell, R.; Kelishadi, R.; Schulin, R. Zinc and its importance for human health: An integrative review. J. Res. Med. Sci. 2013, 18, 144-157.

14. European Commission (EC) on the Provision of Food Information to Consumers, Amending Regulations (EC) No 1924/2006 and (EC) No 1925/2006 of the European Parliament and of the Council, and Repealing Commission Directive 87/250/EEC, Council Directive 90/496/EEC, Commission Directive 1999/10/EC, Directive 2000/13/EC of the European Parliament and of the Council, Commission Directives 2002/67/EC and 2008/5/EC and Commission Regulation (EC) No 608/2004. Available online: https:/ / eur-lex.europa.eu/legal-content/EN/TXT/?uri=CELEX\%3A32011R1169\&qid=1614259348662 (accessed on 25 May 2021).

15. Oria, M.; Harrison, M.; Stallings, V.A. Dietary Reference Intakes for Sodium and Potassium. National Academies of Sciences, Engineering, and Medicine; Health and Medicine Division; Food and Nutrition Board; Committee to Review the Dietary Reference Intakes for Sodium and Potassium; National Academies Press (US): Washington, DC, USA, 2019.

16. Cano-Lamadrid, M.; Girona, D.; García-García, E.; Dominguis-Rovira, V.; Domingo, C.; Sendra, E.; Lopez-Lluch, D.; CarbonellBarrachina, A.A. Distribution of essential and non-essential elements in rice located in a Protected Natural Reserve "Marjal de Pego-Oliva". J. Food Compos. Anal. 2020, 94, 103654. [CrossRef]

17. Punshon, T.; Jackson, B.P. Essential micronutrient and toxic trace element concentrations in gluten containing and gluten-free foods. Food Chem. 2018, 252, 258-264. [CrossRef]

18. Pinto, E.; Almeida, A.; Ferreira, I.M.P.L.V.O. Essential and non-essential/toxic elements in rice available in the Portuguese and Spanish markets. J. Food Compos. Anal. 2016, 48, 81-87. [CrossRef]

19. Antoine, J.M.R.; Hoo Fung, L.A.; Grant, C.N.; Dennis, H.T.; Lalor, G.C. Dietary intake of minerals and trace elements in rice on the Jamaican market. J. Food Compos. Anal. 2012, 26, 111-121. [CrossRef]

20. Joy, E.J.M.; Louise Ander, E.; Broadley, M.R.; Young, S.D.; Chilimba, A.D.C.; Hamilton, E.M.; Watts, M.J. Elemental composition of Malawian rice. Environ. Geochem. Health 2017, 39, 835-845. [CrossRef]

21. Tattibayeva, D.; Nebot, C.; Miranda, J.M.; Cepeda, A.; Mateyev, E.; Erkebaev, M.; Franco, C.M. A study on toxic and essential elements in rice from the Republic of Kazakhstan: Comparing the level of contamination in rice from the European Community. Environ. Geochem. Health 2016, 38, 85-98. [CrossRef] 
22. Rothenberg, S.E.; Mgutshini, N.L.; Bizimis, M.; Johnson Beebout, S.E.; Ramanantsoanirina, A. Retrospective study of methylmercury and other metal(loid)s in Madagascar unpolished rice (Oryza Sativa, L.). Environ. Pollut. 2015, 196, 125-133. [CrossRef]

23. Kashian, S.; Fathivand, A.A. Estimated daily intake of $\mathrm{Fe}, \mathrm{Cu}, \mathrm{Ca}$ and $\mathrm{Zn}$ through common cereals in Tehran, Iran. Food Chem. 2015, 176, 193-196. [CrossRef]

24. Halder, D.; Saha, J.K.; Biswas, A. Accumulation of essential and non-essential trace elements in rice grain: Possible health impacts on rice consumers in West Bengal, India. Sci. Total Environ. 2019, 706, 135944. [CrossRef] [PubMed]

25. Adedire, C.O.; Adeyemi, J.A.; Paulelli, A.C.; da Cunha Martins-Junior, A.; Ileke, K.D.; Segura, F.R.; de Oliveira-Souza, V.C.; Batista, B.L.; Barbosa, F., Jr. Toxic and essential elements in Nigerian rice and estimation of dietary intake through rice consumption. Food Addit. Contam. Part B Surveill. 2015, 8, 271-276. [CrossRef]

26. Ahmed, M.K.; Shaheen, N.; Islam, M.S.; Habibullah-Al-Mamun, M.; Islam, S.; Banu, C.P. Trace elements in two staple cereals (rice and wheat) and associated health risk implications in Bangladesh. Environ. Monit. Assess. 2015, 187, 326. [CrossRef]

27. Prom-u-thai, C.; Rerkasem, B.; Fukai, S.; Huang, L. Key factors affecting Fe density in Fe-fortified-parboiled rice: Parboiling conditions, storage duration, external Fe-loading rate and genotypic differences. Food Chem. 2010, 123, 628-634. [CrossRef]

28. Lee, S.E.; Talegawkar, S.A.; Merialdi, M.; Caulfield, L.E. Dietary intakes of women during pregnancy in lowand middle-income countries. Public Health Nutr. 2013, 16, 1340-1353. [CrossRef]

29. Grygorieva, N.; Povoroznyuk, V.; Romanenko, M.; Synieok, L. Calcium intake in an adult Ukrainian population. Arch. Osteoporos. 2020, 15, 23. [CrossRef]

30. Romero-Marco, P.; Pérez-Gallardo, L.L. Adequacy of calcium intake in Spanish population according age groups. Arch. Osteoporos. 2020, 15, 161. [CrossRef]

31. Jo, G.; Todorov, T.I. Distribution of nutrient and toxic elements in brown and polished rice. Food Chem. 2019, $289,299-307$. [CrossRef]

32. Hensawang, S.; Lee, B.T.; Kimb, K.W.; Chanpiwat, P. Probabilistic assessment of the daily intake of microelements and toxic elements via the consumption of rice with different degrees of polishing. J. Sci. Food Agric. 2020, 100, 4029-4039. [CrossRef] [PubMed]

33. Ortiz, M.L.; Cámara-Martos, F. Bioaccessibility and total content of iron, zinc, copper, an manganese in rice varieties (Oryza sativa L.): A probabilistic assessment to evaluate their contribution to dietary reference intake. Cereal Chem. 2018, 95, 790-799. [CrossRef]

34. Babaali, E.; Rahmdel, S.; Berizi, E.; Akhlaghi, M.; Götz, F.; Mazloomi, S.M. Dietary Intakes of Zinc, Copper, Magnesium, Calcium, Phosphorus, and Sodium by the General Adult Population Aged 20-50 Years in Shiraz, Iran: A Total Diet Study Approach. Nutrients 2020, 12, 3370. [CrossRef] [PubMed]

35. Yamada, M.; Asakura, K.; Sasaki, S.; Hirota, N.; Notsu, A.; Todoriki, H.; Miura, A.; Fukui, M.; Date, C. Estimation of intakes of copper, zinc, and manganese in Japanese adults using 16-day semi-weighed diet records. Asia Pac. J. Clin. Nutr. 2014, $23,465-472$.

36. de Lourdes Samaniego-Vaesken, M.; Partearroyo, T.; Olza, J.; Aranceta-Bartrina, J.; Gil, Á.; González-Gross, M.; Ortega, R.M.; Serra-Majem, L.; Varela-Moreiras, G. Iron Intake and Dietary Sources in the Spanish Population: Findings from the ANIBES Study. Nutrients 2017, 9, 203. [CrossRef]

37. Skolmowska, D.; Głąbska, D. Analysis of Heme and Non-Heme Iron Intake and Iron Dietary Sources in Adolescent Menstruating Females in a National Polish Sample. Nutrients 2019, 11, 1049. [CrossRef]

38. Schiefermeier-Mach, N.; Egg, S.; Erler, J.; Hasenegger, V.; Rust, P.; König, J.; Purtscher, A.E. Electrolyte Intake and Major Food Sources of Sodium, Potassium, Calcium and Magnesium among a Population in Western Austria. Nutrients 2020, $12,1956$. [CrossRef] [PubMed]

39. Choi, M.K.; Bae, Y.J. Dietary Intake and Urinary Excretion of Manganese in Korean Healthy Adults. Biol. Trace Elem. Res. 2020, 196, 384-392. [CrossRef] [PubMed]

40. Filippini, T.; Cilloni, S.; Malavolti, M.; Violi, F.; Malagoli, C.; Tesauro, M.; Bottecchi, I.; Ferrari, A.; Vescovi, L.; Vinceti, M. Dietary intake of cadmium, chromium, copper, manganese, selenium and zinc in a Northern Italy community. J. Trace Elem. Med. Biol. 2018, 50, 508-517. [CrossRef] [PubMed]

41. Waegeneers, N.; Thiry, C.; De Temmerman, L.; Ruttens, A. Predicted dietary intake of selenium by the general adult population in Belgium. Food Addit. Contam. Part A Chem. Anal. Control Expo. Risk Assess. 2013, 30, 278-285. [CrossRef] [PubMed]

42. Wang, Y.; Jia, X.F.; Zhang, B.; Wang, Z.H.; Zhang, J.G.; Huang, F.F.; Su, C.; Ouyang, Y.F.; Zhao, J.; Du, W.W.; et al. Dietary Zinc Intake and Its Association with Metabolic Syndrome Indicators among Chinese Adults: An Analysis of the China Nutritional Transition Cohort Survey 2015. Nutrients 2018, 10, 572. [CrossRef] [PubMed]

43. Zhang, Y.; Liu, Y.; Qiu, H. Association between Dietary Zinc Intake and Hyperuricemia among Adults in the United States. Nutrients 2018, 10, 568. [CrossRef] [PubMed]

44. Chung, M.; Tang, A.M.; Fu, Z.; Wang, D.D.; Newberry, S.J. Calcium Intake and Cardiovascular Disease Risk: An Updated Systematic Review and Meta-analysis. Ann. Intern. Med. 2016, 165, 856-866. [CrossRef]

45. Fassier, P.; Egnell, M.; Pouchieu, C.; Vasson, M.P.; Cohen, P.; Galan, P.; Kesse-Guyot, E.; Latino-Martel, P.; Hercberg, S.; Deschasaux, M.; et al. Quantitative assessment of dietary supplement intake in 77,000 French adults: Impact on nutritional intake inadequacy and excessive intake. Eur. J. Nutr. 2019, 7, 2679-2692. [CrossRef] [PubMed]

46. Shakur, Y.A.; Tarasuk, V.; Corey, P.; O'Connor, D.L. A comparison of micronutrient inadequacy and risk of high micronutrient intakes among vitamin and mineral supplement users and nonusers in Canada. J. Nutr. 2012, 142, 534-540. [CrossRef] 